\title{
Anabases
}

ANABASES Traditions et réceptions de l'Antiquité

12 | 2010

Varia

\section{Interpreti francesi di Isocrate: Clermont-Tonnerre}

\section{Pasquale Massimo Pinto}

\section{OpenEdition}

\section{Journals}

Edizione digitale

URL: http://journals.openedition.org/anabases/1234

DOI: 10.4000/anabases.1234

ISSN: 2256-9421

\section{Editore}

E.R.A.S.M.E.

\section{Edizione cartacea}

Data di pubblicazione: 1 ottobre 2010

Paginazione: 208-215

ISSN: 1774-4296

\section{Notizia bibliografica digitale}

Pasquale Massimo Pinto, «Interpreti francesi di Isocrate: Clermont-Tonnerre », Anabases [Online], 12 | 2010, Messo online il 01 octobre 2013, consultato il 20 octobre 2019. URL : http:// journals.openedition.org/anabases/1234 ; DOI : 10.4000/anabases.1234

Questo documento è stato generato automaticamente il 20 ottobre 2019.

(c) Anabases 


\title{
Interpreti francesi di Isocrate: Clermont-Tonnerre
}

\author{
Pasquale Massimo Pinto
}

La ricezione di Isocrate in età moderna costituisce un ambito molto ricco e ancora in buona parte da esplorare. Come per molti degli autori antichi importanti, soprattutto quelli la cui produzione si è più facilmente prestata nel tempo a letture politiche $\mathrm{e}$ variamente ideologiche, anche nel caso di Isocrate sarebbe interessante tracciare la storia della sua fortuna nelle diverse culture europee ${ }^{1}$. Come piccolo contributo in questa direzione, può essere utile ricordare qui l'opera, largamente dimenticata, di un singolare interprete di Isocrate nella Francia del secondo Ottocento.

2 1. Tra il 1862 e il 1864 uscirono a Parigi, presso Didot, tre corposi ed eleganti volumi in ottavo, contenenti la traduzione francese dell'intero corpus delle opere di Isocrate $^{2}$ : i ventuno opuscoli ed orazioni, più le nove lettere ${ }^{3}$, accompagnati da un dettagliato sommario del contenuto, e seguiti dai frammenti, dalle hypotheseis dei discorsi (in greco), e da un ricco indice di nomi e cose. Tutta l'opera era inoltre introdotta da un beve ma denso avant-propos (p.v-vii). Autore della notevole impresa, che aveva richiesto un trentennale periodo di gestazione, non era stato né un filologo professionista, né un classicista o un letterato, bensì un ex militare e uomo politico da tempo ormai lontano dalla vita pubblica: l'ottuagenario duca Aimé-Marie-Gaspard de Clermont-Tonnerre (1779-1865).

3 Nato da una famiglia dell'antica nobiltà francese, Clermont-Tonnerre si era preparato alla carriera militare attraverso gli studi all'École polytechnique di Parigi. Nell'istituto creato dal Bonaparte era tornato come insegnante, per poi servire a lungo in armi durante le campagne napoleoniche in Italia e in Spagna come ufficiale. Con la Restaurazione incominciò la sua ascesa politica: dopo essere stato chiamato nel 1815 alla neo-istituita «Chambre des Pairs», durante i regni di Luigi XVIII e Carlo X fu due volte ministro nel governo di Villèle, della marina dal 1821 al 1824 e della guerra dal 1824 al 1828. Durante quest'ultimo mandato ideò il progetto dell'occupazione di Algeri, poi realizzata nel 1830. La rivoluzione del luglio 1830 e il rifiuto di giurare fedeltà al 
nuovo regime di Luigi Filippo segnarono il suo allontanamento dalla politica e il ritiro a vita privata nella tenuta di Glisolle, nel distretto di Evreux, in Normandia ${ }^{4}$.

4 La notorietà di Clermont-Tonnerre nel campo degli studi classici, una notorietà conquistata solo negli ultimissimi anni di vita, è unicamente legata alla traduzione di Isocrate ${ }^{5}$. Si può affermare anzi che essa sia l'opera di Clermont-Tonnerre, quella che lo impegnò per il maggior tempo e che, pur attraverso la mediazione di un autore antico, meglio rappresenta l'uomo'.

5 Clermont-Tonnerre aveva imparato il greco da adulto, spinto dal desiderio di leggere nell'originale i testi della letteratura antica. Il suo primo biografo, Émile Egger, racconta che la decisione di imparare il greco era maturata in occasione di una missione compiuta nelle isole ionie nel 1807, e ci dà anche il nome di colui che fu il suo maestro, Gregorios Georgiades Zalyk ${ }^{7}$, uno studioso di Salonicco trapiantato a Parigi, ben noto negli ambienti della diplomazia per aver lavorato a lungo come interprete. Zalyk gli aveva insegnato a pronunciare il greco antico secondo la pronuncia bizantina, pronuncia a cui l'allievo si era poi attenuto fino alla fine della sua vita. Da quel momento Clermont-Tonnerre aveva letto diversi autori (tra cui Tucidide, Senofonte e Demostene) e tra le sue carte si ritrovano, come segnalò Egger, numerose testimonianze della conoscenza di autori greci. Isocrate divenne però indubbiamente l'autore della sua vita, o meglio della sua maturità e vecchiaia.

6 La lettura di Isocrate di Clermont-Tonnerre si inserisce in una linea conservatrice e tradizionale consolidata, che dell'opera dello scrittore ateniese ha privilegiato per secoli gli aspetti patriottici e morali. Per un nobile francese, cattolico e legittimista, già militare e uomo di governo, l'opera - vasta eppure organica e coerente - di un autore critico verso la democrazia ateniese, ma glorificatore della propria patria e sereno moralista, costituiva una confortante palestra di riflessione negli anni della maturità e della vecchiaia. Nella sua sobrietà, già l'avant-propos delinea con chiarezza lo stretto legame tra impegno letterario e ragioni biografiche. Nonostante la cesura segnata dal luglio 1830 - l'«ora del sacrificio» nelle parole di Clermont-Tonnerre - l'attività letteraria è presentata come una prosecuzione dell'impegno politico: «La situation des affaires me fit pressentir que l'heure du sacrifice s'approchait; elle sonna en effet pou moi en 1830. Dans des telles circonstances, l'étude des lettres ne vient pas seulement remplir nos loisirs et accroître nos facilités: elle nous offre encore la consolante pensée de pouvoir être utiles à nos semblables en dehors des luttes politiques qui les agitent, et à notre patrie, en soutenant les doctrines qui font le bonheur et la grandeur des peuples ${ }^{8} . »$

7 Nella prefazione, la scelta di dedicarsi all'«étude des lettres» e a un autore come Isocrate è motivata principalmente con l'utilità che può derivarne per il prossimo e per la patria. Elencando i «meriti» dell'autore scelto, Clermont-Tonnerre mette in rilievo innanzi tutto che dall'inizio della sua carriera fino all'estrema vecchiaia Isocrate, benché privo di una voce adatta per parlare in pubblico ${ }^{9}$, poté tuttavia produrre opere che contribuirono al bene e all'onore di Atene. Fa seguire poi una valutazione estetica molto elogiativa: Isocrate può essere collocato al primo posto tra gli oratori di tutti i paesi e di tutti i secoli per l'abilità nel maneggiare una lingua estremamente ricca e armoniosa. E, infine, di gran parte dell'opera di Isocrate sottolinea il carattere morale e l'affinità con il cristianesimo, con la «lumière de l'Évangile ${ }^{10}$ ».

8 La realizzazione di una versione francese adatta alle esigenze e al gusto dell'epoca, ed efficace nel rendere il carattere della prosa isocratea, comportò indubbiamente un 
forte impegno linguistico e stilistico. Per quanto riguarda gli apparati informativi, però, l'opera di Clermont-Tonnerre fu prevalentemente una operazione compilativa, fondata su erudizione e studi precedenti, cosa che non sorprende date la formazione del curatore e la condizione "amatoriale» in cui il lavoro fu svolto, nonostante l'autore fosse confortato dal consiglio di studiosi professionisti ${ }^{11}$. Clermont-Tonnerre si servì comunque di strumenti per l'epoca aggiornati: l'edizione degli Oratores Attici di Carl Müller (Paris, Didot, 1846) e quella isocratea di Gustav Eduard Benseler (Leipzig, Teubner, 1851-1852), soprattutto per il testo ${ }^{12}$; la precedente edizione francese di tutto Isocrate, quella greco-latina curata dall'abate Athanase Auger (Paris, Didot, 1782), dalle cui introduzioni egli trasse diverse pagine per i suoi Arguments ${ }^{13}$; e quella pubblicata da Wilhelm Lange (Halle, Libraria Hemmerdeana, 1803), da cui tradusse i sommari. Talora fece ricorso anche alla vecchia edizione di Hieronymus Wolf (Basel 1570), rimasta in uso per secoli ${ }^{14}$. Non sono invece mai citati studi, tranne un lungo estratto da una memoria di Egger sul mestiere di avvocato e logografo a Atene ${ }^{15}$.

9 L'impostazione tradizionale e la natura compilativa di gran parte dell'opera non impediscono tuttavia di individuare alcuni apporti personali del curatore, talora non privi di originalità o interesse. Significativa, a questo proposito, è la lettura «legittimista» del Nicocle, discorso scritto per il sovrano di Salamina di Cipro in cui Nicocle parla ai suoi concittadini dei doveri del re e dei sudditi. L'introduzione di Clermont-Tonnerre si diffonde sul valore della monarchia ereditaria, sulla superiorità della monarchia rispetto alla repubblica e sulla conservazione delle grandi famiglie che contribuiscono al prestigio dello stato: «On est donc autorisé à penser que c'est du pouvoir monarchique héréditaire que veut parler Isocrate, quand il traite des droits et des devoirs des rois; et la comparaison qu'il fait du gouvernement d'un seul avec les gouvernements multiples, en montrant la supériorité de la monarchie, dans la guerre comme dans la paix, prouve à quel point il avait su apprécier les avantages de la force morale que donne l'hérédité à l'unité monarchique. [...] Isocrate insiste encore sur un autre point d'une haute importance: c'est la conservation des familles dont l'illustration est une partie des gloires du pays. Les sentiments élevés sont la conséquence d'une noble origine, et sous peine de déshonneur, on les exige de ceux que le hasard de leur naissance a privilégiés ${ }^{16}$ ». I lunghi trascorsi nell'amministrazione e nel governo fanno guardare Clermont-Tonnerre con particolare interesse ad alcuni aspetti antiquari: l'Argument dell'Antidosi, ad esempio, si apre con una esposizione del sistema delle liturgie che consentiva ad Atene di garantirsi, attraverso la contribuzione dei cittadini più ricchi, alcuni servizi, in particolare l'equipaggiamento di una flotta attraverso la trierarchia ${ }^{17}$. Né mancano osservazioni su ambiti ancora poco esplorati all'epoca, come quella sul concetto di proprietà letteraria nel mondo antico, «chose beaucoup moins déterminée que sous le règne de nos législations modernes», formulata a proposito del corpuscolo epistolare isocrateo e in relazione al problema della formazione delle collezioni di lettere di autori antichi ${ }^{18}$. $O$, ancora, è interessante notare che sia presentato in tono apologetico un opuscolo come l'Elena, testo che poteva apparire discordante con l'immagine dell'autore antico che Clermont-Tonner cercava di ricostruire: «Ce discours est regardé comme l'un des plus remarquables d'Isocrate; mais on comprend que, la nature du sujet étant peu en harmonie avec la sévère morale qui est un des traits caractéristiques des oeuvres du grand orateur, il ait été l'objet de beaucoup de critiques $^{19}$.» Ad una analoga preoccupazione rispondeva in sostanza anche il tentativo, sulla scorta di Auger, di ridurre il numero dei discorsi giudiziari da considerare effettivamente scritti per occasioni reali ${ }^{20}$. 
10 2. A Clermont-Tonnerre come interprete di Isocrate dedicò uno studio uno dei più autorevoli e influenti classicisti francesi della seconda metà del XIX secolo, Émile Egger $(1813-1885)^{21}$. Si tratta di un lavoro interessante per diversi aspetti, non ultimo per la sua articolata vita editoriale. Egger era legato a Clermont-Tonnerre da un rapporto d'amicizia che datava dal 1857 ed era stato uno di coloro che ne avevano seguito da vicino il lavoro di traduttore ${ }^{22}$. Subito dopo la morte del duca, aveva pubblicato sul periodico Le Correspondant un ampio articolo dal titolo Le duc de Clermont-Tonnerre traducteur et commentateur des cuvres d'Isocrate ${ }^{23}$. Nello stesso 1865 il saggio fu pubblicato separatamente in forma di opuscolo: Notice historique sur le duc de ClermontTonnerre traducteur et commentateur des cuvres d'Isocrate, par E. Egger, Membre de l'Institut, Paris, Chez Ad. Lainé, Libraire-éditeur, $1865^{24}$. Il ritratto di un ex-generale ed ex-ministro legittimista, «retenu loin de la vie politique depuis 1830 par d'honorables scrupules de conscience ${ }^{25}$ » e dedito allo studio dei classici, dovette suscitare un certo interesse nella società letteraria e politica della Francia del Secondo Impero: nel 1866 apparve infatti, presso il medesimo editore, una seconda edizione, la cui pubblicazione, come dichiara l'autore, era stata decisa in seguito alle richieste dei lettori, dopo che la tiratura era andata esaurita ${ }^{26}$. La nuova edizione, accresciuta di due pagine e qualche rigo, era preceduta da un «Avertissement» datato «Octobre 1866» contenente ulteriori informazioni biografiche su Clermont-Tonnerre ${ }^{27}$. Nel 1868 la Notice riapparve ancora una volta, in terza edizione, in una ristampa che si distingueva soltanto per la presenza di una Appendice in cui Egger riproponeva le pagine che aveva dedicato all'opera di Clermont-Tonnerre subito dopo l'uscita del III volume ${ }^{28}$. La vicenda editoriale di questo opuscolo non si esaurisce però qui: Clermont-Tonnerre doveva essere un soggetto interessante anche per un certo pubblico dell'Inghilterra vittoriana, visto che nel frattempo, nel 1867, Egger aveva ritenuto utile dare alle stampe, sempre a Parigi, anche una traduzione inglese della seconda edizione ${ }^{29}$.

11 L'idea che attraversa tutto l'opuscolo di Egger è la forte affinità tra l'autore antico e il suo interprete moderno, una serie di tratti comuni che farebbero di quest'ultimo una specie di Isocrate francese ma cristiano: «il semblait que chaque événement de notre vie publique, chaque nouveau devoir que la famille, que l'amitié, que la patrie lui venait apporter, le ramenât vers son auteur favori. Tout lui était une raison nouvelle d'apprécier les conseils du moraliste $\operatorname{grec}^{30}$.» Una affinità che, implicitamente, Clermont-Tonnerre stesso aveva per primo voluto riconoscere.

12 Ma l'aspetto che rende particolarmente prezioso il lavoro di Egger è la documentazione inedita che Egger poté utilizzare. Ebbe infatti la possibilità di lavorare sulle carte di Clermont-Tonnerre subito dopo la sua morte. Questi materiali erano all'epoca conservati nel castello di Glisolles, dove il duca aveva vissuto l'ultima parte della sua $v$ ita $^{31}$. L'esame degli autografi condotto da Egger dimostrava che nel piano originario della sua opera Clermont-Tonnerre aveva previsto anche un commento, progetto intrapreso e parzialmente realizzato. Se il progetto fosse stato portato a compimento, Clermont-Tonnerre avrebbe dato vita probabilmente a un lavoro notevole che non aveva precedenti, un commento all'intera opera di Isocrate. Egger dichiara di aver esaminato un manoscritto non ultimato, lasciato in una redazione provvisoria, con parti più e meno rifinite, dal quale è in grado di citare alcuni stralci, che ci danno un'idea dell'impostazione che Clermont-Tonnerre avrebbe dato alle sue note. Possiamo, in conclusione, riportare alcune delle osservazioni di Egger: «En général, la méthode de l'helléniste homme d'État n'a jamais varié. [...] c'est partout le même soin à interroger 
l'histoire, bien entendu celle des peuples classiques, depuis la Bible jusqu'à Mézeray, pour y trouver des arguments en faveur des vérités qui doivent diriger la conduite des homes d'État. Le sujet qui revient le plus souvent dans le commentaire sur Isocrate est celui que je retrouve à chaque page des écrits antérieurs, de ceux qui étaient le moins destinés à l'impression: je veux dire la théorie d'une monarchie fondée sur le respect de la tradition, la théorie religieuse et politique de Bossuet, avec quelques amendements suggérés par les leçons du temps. Sur l'autorité du peuple et du vote populaire, en matière de gouvernement, les convictions du commentateur sont celles d'un politique libéral, mais dont le libéralisme faisait peu de concessions à l'esprit des sociétés modernes $^{32} . »$

\section{NOTE}

1. Uno studio importante in questo senso è quello di Lucia GUALDO ROSA, La fede nella «paideia». Aspetti della fortuna europea di Isocrate nei secoli XV e XVI, Roma, 1984.

2. Cuvres complètes d'Isocrate. Traduction nouvelle avec texte en regard par le Duc de Clermont-Tonnerre (Aimé-Marie Gaspard), ancien ministre de la Guerre et de la Marine, Ancien élève de l'École polytechnique, Paris, Librairie de Firmin Didot Frères, Fils et C.ie [...], I (1862), vii + 469 pp.; II (1863), 475 pp.; III (1864 [solo questo volume: «et chez Auguste Durand, Libraire [...]»), $624 \mathrm{pp}$.

3. Anzi dieci, dal momento che l'edizione includeva - nonostante Clermont-Tonnerre fosse consapevole della falsa attribuzione (Euvres complètes d'Isocrate, III, p. 465) - anche la lettera fittizia di Teofilatto Simocatta (VII d.C.) a Dionisio di Siracusa (79 Zanetto), stampata per secoli, a partire dall'edizione aldina di Isocrate del 1534, tra le lettere isocratee.

4. Due brevi profili biografici, tra loro collimanti, pubblicati quando ClermontTonnerre era ancora in vita si leggono nel Dictionnaire de la conversation et de la lecture, Seconde édition, V, Paris, 1853, p. 727-728, e nella Nouvelle Biographie générale dello Hoefer, X, Paris 1856, coll. 844-845. Si veda ora É. FRANCESCHINI, in Dictionnaire de biographie française, VIII, Paris, 1959, coll. 1514-1515. Un più articolato e significativo profilo, pubblicato all'indomani della morte e dell'uscita del terzo volume dell'Isocrate, e incentrato sulla sua opera di studioso, si deve all'amico Émile EGGER, Notice historique sur le duc de Clermont-Tonnerre (Paris, 1865): su quest'opera si dirà nel dettaglio più oltre. Una biografia analitica apparirà solo venti anni dopo la morte; si tratta dell'importante lavoro, fondato anche sulle carte private del biografato, di uno storico del Ministero della guerra francese: Camille ROUSSET, Un ministre de la Restauration. Le marquis de Clermont-Tonnerre, Paris, 1885 (per la traduzione di Isocrate si vedano le p. 434-435). 5. Pare che in gioventù avesse tradotto dal tedesco una "storia della costituzione polacca dell 3 maggio 1791" di Stanislaw Potocki per le ricerche di A.-C.-F. Ferrand sulla storia polacca (EGGER, Notice historique sur le duc de Clermont-Tonnerre, p. 10 n. 1). Non molto altro fu da lui pubblicato durante gli anni del suo ritiro: possiamo ricordare la breve Notice biographique dedicata a Élie-Louis-Aymar, Marquis de Dampierre, Paris, impr. de Ducessois, 1845; e la cura per una seconda edizione di un'opera di un altro Pari di 
Francia, il conte Antoine-François-Claude Ferrand (di cui aveva pronunciato alla Camera dei Pari l'elogio funebre nel 1825): Éloge historique de Madame Élisabeth de France, suivi de plusieurs lettres de cette princesse, Paris, impr. de A. Le Clère, 1861.

6. I volumi della traduzione ebbero in generale una buona accoglienza. Si veda ad esempio la recensione di Désire NISARD apparsa sul Moniteur del 9 novembre 1863, poi raccolta nelle sue Nouvelles Études d'histoire et de littérature, Paris, 1864, p. 313-329 (la raccolta di Nisard contiene anche altri saggi particolarmente interessanti per gli antichisti: Démosthènes, orateur politique, Une des campagnes de Jules César dans les Gaules e Un philologue hollandais: M. Cobet). É. EGGER recensì Clermont-Tonnerre sul Journal des Savants del 1864, all'interno di un saggio di recensione di recenti edizioni di oratori, alle p. 442-455. Un giudizio positivo di J.F. BOISSONADE si legge in Critique littéraire sous le premier empire [ed. F. Colincamp], I, Paris, 1863, p. 430 n. 2. Si vedano anche ANOT DE MAIZIÈRE, Bibliographie Catholique 29 (1863), p. 209-213 e la lunga recensione compresa all'interno di un ampio saggio di Pierre Willems sulla Revue de l'Instruction publique en Belgique 12 (n.s. 7) (1864) alle p. 369-381 e 389-405. Non mancò qualche voce discordante, come ad esempio quella di Georges PERROT, L'éloquence politique et judiciaire à Athènes, I. Les précurseurs de Démosthène, Paris, 1873, p. 286 n. 1: «Le papier et l'impression sont superbes; le français est pénible.»

7. Cfr. Notice historique sur le duc de Clermont-Tonnerre, p. 10-11. Nato a Salonicco nel 1785, morto a Parigi nel 1827, Zalyk fu l'autore di un apprezzato Dictionnaire français et grec moderne (Paris, 1809), e traduttore in greco del Contrat social di Rousseau (lavoro pubblicato postumo, Paris, 1828). Collazionò manoscritti greci della «Bibliothèque imperiale» per diversi filologi europei e curò alcune edizioni di testi, p. es.: il discorso Sulla libertà dei Rodii di Demostene (Paris, Mme Aumont, 1815) e la Vita di Mario di Plutarco (Paris, Mme Aumont Vve Nyon, 1815; impr. d'Eberhard, 1816). Scrisse anche un Dialogue sur la révolution grecque (1829) a cui il suo sodale e biografo Constantinos Nicolopoulos premise un Discours adressé à tous les jeunes Grecs sur l'importance de la littérature et de la philosophie grecques. Su di lui cfr. la voce di C. NIcolopoulos in Biographie universelle ancienne et moderne (Michaud), 52, Paris, 1828, p. 65-66; e Ch. M.W. BRUNET DE PRESLE, "M. Hase et les savants grecs émigrés à Paris sous le premier empire et sous la restauration", Revue des cours littéraires 2 (1865), p. 317-326: 323-324.

8. Euvres complètes d'Isocrate, I, p. v-vi.

9. Clermont-Tonnerre accoglie la versione autobiografica (cfr. Filippo 81, Panatenaico $9 \mathrm{e}$ 10) divenuta poi topica nella tradizione biografica (Pseudo-Plutarco, Vite dei dieci oratori 837A, 838DE; Filostrato, Vite dei sofisti 505 Kayser; Vita anonima rr. 35-36 MathieuBrémond). Sulla «retorica» del non poter parlare in pubblico cfr. Y.L. Too, The Rhetoric of Identity in Isocrates. Text, Power, Pedagogy, Cambridge, 1995, p. 74-112. Ma cfr. già M. SILVESTRINI, “Terminologia politica isocratea. II. L'«Areopagitico» o dell'ambiguità isocratea", Quaderni di storia 7 (1978), p. 169-183: 180 n. 1.

10. La «cristianizzazione» dell'Isocrate moralista cominciò abbastanza presto ed ebbe ampia diffusione nella cultura tardoantica e bizantina. Un manoscritto del xv secolo, il Laurenziano 74.13, in coda all'A Demonico conserva un tristico che proclama anacronisticamente la perfezione di Isocrate, se solo avesse creduto nell'unico dio dei cristiani, cfr. E. DRERUP, Isocratis opera omnia, I, Leipzig, 1906, p. XXIII. Sulla religiosità di Isocrate cfr. da ultimo J.A.E. BONS, "Isocrates on being religious and moral conduct", in A.P.M.H. LARDINOIS - M.G.M. VAN DER POEL - V.J.C. HUNINK (eds.), Land of Dreams. Greek and Latin Studies in Honour of A.H.M. Kessels, E.J. Brill, Leiden-Boston, 2006, p. 259-266, che 
ricorda nella chiusa del saggio proprio le parole dell'introduzione di ClermontTonnerre.

11. ROUSSET, Un ministre de la Restauration: le marquis de Clermont-Tonnerre, p. 434, segnala che dalle prime fasi del lavoro Clermont-Tonnerre fu coadiuvato dal consiglio del filologo e orientalista Adolphe Regnier (1804-1884) e di Egger.

12. Cfr. Cuvres complètes d'Isocrate, III, p. 547.

13. In alcuni casi riprese interamente l'introduzione di Auger, come nel caso del Busiride, cfr. CEuvres complètes d'Isocrate, II, p. 200-201.

14. Cfr. p. es. CEuvres complètes d'Isocrate, I, p. 418.

15. Cfr. Euvres complètes d'Isocrate, III, p. 265-266. Lo studio, intitolato Si les Athéniens ont connu la profession d'avocat, fu presentato alla Academie des inscriptions et belles-lettres il 7 dicembre 1860, e poi pubblicato nei Mémoires de littérature ancienne (Paris, 1862), p. 355-388.

16. Cfr. Cuvres complètes d'Isocrate, I, p. 74-75.

17. Oeuvres complètes d'Isocrate, III, p. 1. Si noti a questo proposito che l'edizione di Clermont-Tonnerre conteneva anche la prima traduzione francese del testo integrale dell'Antidosi, riscoperto da Andrea Mustoxydis nel 1812. Nel 1862 era uscita sempre a Parigi l'edizione con commento dell'Antidosis a cura di Ernest Havet, che includeva la traduzione realizzata da Auguste Cartelier. Il volume di Clermont-Tonnerre contenente l'Antidosi apparve nel 1864, ma si dovrà prestar fede all'autore quando afferma che non era al corrente della contemporanea operazione di traduzione. Lo dichiara in un post scriptum dell'Argument dell'orazione: «Cet argument était écrit et déjà livré aux imprimeurs, lorsque nous avons reçu de M. Ernest Havet une publication dont voici le titre [...]. Cette publication, qui est d'un grand intérêt, aura ainsi précédé la nôtre de quelques mois seulement» (Euvres complètes d'Isocrate, III, p. 4).

18. CEuvres complètes d'Isocrate, III, p. 464.

19. Cfr. Cuvres complètes d'Isocrate, II, p. 156-157.

20. Cfr. Euvres complètes d'Isocrate, III, p. 264-265.

21. Su Egger cfr. A. BAILly, Notice sur Émile Egger [...] sa vie et ses travaux, Paris, 1886. Da ultimo, P. HUMMEL, "Émile Egger, historiographe des mutations philologiques", L'Antiquité Classique 75 (2006), p. 155-160.

22. Notice historique sur le duc de Clermont-Tonnerre, p. 6-7. Cfr. anche supra la n. 11.

23. "Le duc de Clermont-Tonnerre traducteur et commentateur des œuvres d'Isocrate", Le Correspondant 65 (n.s. 29), (1865) p. 830-847.

24. L'opuscolo riprendeva in copertina il medesimo motivo ornamentale che appariva sulla copertina dei volumi di Clermont-Tonnerre. Tra il testo della prima edizione della Notice e quello dell'articolo apparso sul Correspondant c'è una sola piccola differenza, l'aggiunta di una nota a p. 10 della Notice.

25. É. EGGER, Journal des Savants, luglio 1864, p. 442-455: 447.

26. Egger dichiara di aver voluto ripubblicare l'opuscolo per rispondere alle richieste di «quelques amis des lettres et de l'histoire» e per rinnovare il piacere di «un commerce intime avec l'éminent homme de bien que nous avons voulu peindre» (p. 1-2).

27. L'intervento più consistente si legge alle p. 26-28, una sezione dedicata al riconoscimento del genio e dello stile isocratei - ma ancora nel testo della terza edizione si legge il fatale refuso «socratiques» - nei pochi scritti politici dati alle stampe da Clermont-Tonnerre. Forse con eccesso di enfasi, Egger così chiosava la rievocazione di un discorso tenuto da Clermont-Tonnerre nel 1819 alla Camera dei Pari in merito all'abolizione del «droit d'aubaine»: «Si Athènes avait eu jamais un Sénat, au sens 
moderne de ce mot, une Chambre des pairs, Isocrate y aurait pu obtenir des succès comme celui-là» (p. 28). Per il testo dell'intervento di Clermont-Tonnerre cfr. La législation civile, commerciale et criminelle de la France, ou commentaire et complément des codes français [...] par M. le baron Locré, X, Paris, 1827, p. 334-353.

28. Notice historique sur le duc de Clermont-Tonnerre ${ }^{3}$, p. 49-52 = Journal des Savants, luglio 1864, p. 447-449 (all'interno di una recensione cumulativa di lavori dedicati agli oratori attici).

29. Historical notice on the Duke de Clermont-Tonnerre translator and commentator of the works of Isocrates by E. Egger Member of the Institute, Paris, Ad. Lainé, Printer and Publisher, 1867 (48 p.). Nell'edizione inglese non c'è nulla che illustri le ragioni alla base di questa operazione editoriale.

30. Notice historique sur le duc de Clermont-Tonnerre, p. 38. E già nella recensione apparsa sul «Journal des Savants» Egger scriveva: «Isocrate l'attirait d'un charme particulier, grâce à l'élévation soutenue de sa morale, si voisine parfois de l'Évangile, un peu aussi par les caractères de sa politique, politique honnête et si sévère aux excès de la démocratie qu'elle semble parfois incliner aux préférences monarchiques et préparer les Grecs à la domination des rois de Macédoine» (p. 447 = Notice historique sur le duc de Clermont-Tonnerre ${ }^{3}$, p. 50).

31. Parte delle carte personali di Clermont-Tonnerre si trova ora nelle Archives Nationales (359 AP 118): da segnalare le "Notes manuscrites sur la 'guerre de Julius Caius Cesar à Alexandrie”". Nelle Archives départementales de l'Eure, Autographes «La Sicotière» $35 \mathrm{~J} 8$, si conservano sue lettere.

32. Notice historique sur le duc de Clermont-Tonnerre, p. 25-26.

\section{AUTORE}

\section{PASQUALE MASSIMO PINTO}

Dipartimento di Scienze dell'antichità

Università di Bari

m.pinto@lettere.uniba.it 